PALABRAS CLAVE

Competitividad

Desarrollo regional

Medición

Gobernabilidad

Desarrollo económico

Productividad

Recursos humanos

Administración de empresas

Jorge Benzaquen

Profesor de CEnTRum Católica, Pontificia Universidad Católica del Perú

\section{• ibenzaq@pucp.edu.pe}

Luis Alfonso del Carpio

Profesor de CENTRUM Católica, Pontificia Universidad Católica del Perú

○ldelcarpio@pucp.edu.pe

Luis Alberto Zegarra

Profesor de CENTRUM Católica, Pontificia

Universidad Católica del Perú

cogarra.la@pucp.edu.pe

Christian Alberto Valdivia

Profesor de CENTRUM Católica, Pontificia

Universidad Católica del Perú

•cvaldiviao@pucp.edu.pe
REVISTA CEPAL 102 DICIEMBRE 2010

\section{Un Índice Regional de Competitividad para un país}

\author{
Jorge Benzaquen, Luis Alfonso del Carpio, \\ Luis Alberto Zegarra y Christian Alberto Valdivia
}

$\mathrm{E}$

el presente trabajo se presenta una propuesta para el desarrollo de un Índice de Competitividad Regional de un país que forma parte de una investigación integral de medición del nivel de competitividad. Con esta propuesta se aspira a posicionar un nuevo enfoque para la competitividad, al medir cómo en determinada región de un país se administran los recursos y capacidades para incrementar sostenidamente la productividad empresarial y el bienestar de su población. Se identificaron los determinantes de la competitividad de las regiones, denominándolos pilares: i) gobierno e instituciones; ii) desarrollo económico; iii) infraestructura productiva; iv) capital humano, y v) eficiencia de las empresas. Para cada uno de ellos se identificaron cinco factores y sus variables con que se miden diversos aspectos de la competitividad regional. Estos constituyen un segundo y tercer nivel de desagregación que aportan al análisis que se puede realizar con los resultados que se obtengan. 


\section{I \\ Introducción}

La competitividad de las regiones que constituyen un país juega un papel importante para que los esfuerzos se orienten a lograr un desarrollo sostenido que incida en el bienestar de la población. La medición de la competitividad de las regiones ha sido abordada en diversos estudios, en que se recogen algunos de los componentes necesarios para desarrollar una propuesta metodológica de medición.
En este artículo se presenta una propuesta para el desarrollo de un Índice de Competitividad Regional (ICR) de un país, como parte de una investigación integral de medición del nivel de competitividad.

Con ella se aspira a posicionar un nuevo enfoque para la competitividad, midiendo cómo en determinada región de un país se administran los recursos y capacidades a objeto de incrementar sostenidamente la productividad empresarial y el bienestar de su población.

\section{II}

\section{La competitividad}

El concepto de competitividad no es reciente, sus orígenes se remontan a más de tres siglos atrás con las teorías de comercio. Sin embargo, hoy en día no existe consenso sobre su definición y, por ende, acerca de una forma de medirla.

El concepto de ventaja competitiva se fundamenta en la "productividad" y en los factores que la determinan dentro de la empresa. La productividad es la relación entre la producción obtenida mediante un sistema de producción de bienes y servicios y los recursos utilizados para obtenerla, es decir, su uso eficiente o la relación entre los resultados obtenidos con respecto de los recursos usados y el tiempo que toma conseguirlos (D’Alessio, 2004, pág. 223). Este enfoque, ampliamente aceptado desde la perspectiva de los factores que la componen, es presentado por Prokopenko en 1972 en Productivity Managment, A Practical Handbook (Prokopenko, 1972).

Esta visión de la productividad se enriqueció en los años ochenta del siglo pasado con la amplia divulgación de la obra Estrategia competitiva: técnicas para el análisis de los sectores industriales y de la competencia (Porter, 2000), donde se propone un modelo para entender a las industrias y la competencia, así como para formular una estrategia global. En el modelo se presentan los cinco factores de la competencia que determinan el atractivo de un sector industrial y sus causas, así como los que cambian con el tiempo y pueden ser modificados mediante la estrategia.
Porter (1999) plantea el enfoque de ventaja competitiva como el valor que una empresa logra crear para sus clientes, y que supera los costos. Este valor corresponde a lo que los individuos están dispuestos a pagar y alcanza su expresión superior en la medida que los precios de oferta son más bajos que los ofrecidos por la competencia. Para ello se requiere que las personas obtengan beneficios equivalentes. La estrategia óptima debiera reflejar una adecuada comprensión del entorno de la empresa.

Si bien es cierto que el origen del concepto de competitividad de una nación se remonta a varios siglos atrás con las teorías de comercio, es Porter (1991) en La ventaja competitiva de las naciones quien establece las bases y reconoce los cambios en el entorno y la inestabilidad de las estrategias genéricas, señalando la necesidad de contar con modelos más dinámicos para concebir la ventaja competitiva de las naciones.

Asimismo, Krugman (1994) sostiene que la competitividad pierde relevancia en el ámbito nacional, ya que los principales países no están compitiendo entre ellos, por lo que se trata más bien de un asunto interno de la nación que de un aspecto externo. Al respecto, Porter (1991) afirma que la competitividad de una nación depende de la capacidad de sus industrias para innovar y mejorar, y que determinadas empresas son capaces de hacerlo con coherencia, procurando denodadamente las mejoras y una fuente cada vez más perfeccionada de ventaja competitiva. 
Camagni (2005), en su libro Economía urbana, destaca el debate derivado de la posición de Paul Krugman (1998, pág. 5) con respecto a la competitividad internacional, que pone en duda la idea de que la prosperidad de un país depende de su éxito comercial.

Para Camagni (2005), el principio de la ventaja comparativa no puede aplicarse cuando se analiza la competitividad entre economías locales y de comercio interregional, debido a que esta teoría se basa en el concepto de costos-precios relativos que se ajustan motivados por la no movilidad de factores productivos, la devaluación de la moneda y la flexibilidad a la baja de precios y salarios dada la situación autárquica o de aislamiento. Sin embargo, cuando hablamos de economías regionales existen factores que desvían el principio de ventaja comparativa de sus fundamentos:

i) no se puede hablar de autarquía o aislamiento: se pierde el vínculo entre productividad media y salarios reales;

ii) existen movimientos de factores productivos entre regiones: una región que registra una desventaja absoluta en todos los bienes va a presentar desequilibrios en el mercado laboral debido a la movilidad de factores. Esta región se ajusta más rápidamente mediante la emigración y el despoblamiento que por medio de ajuste de precios, y iii) no existe una moneda regional o un tipo de cambio específico para cada territorio: a partir de una situación de equilibrio en que cada región presenta una ventaja absoluta en algún producto, si alguna región ve que su productividad aumenta en menor medida que la de las otras regiones y que su producto pierde competitividad no podría utilizar una herramienta con que cuentan los países, como la devaluación de su moneda. En síntesis, la evolución de la teoría de la competitividad parte de dos teorías fundamentales: la teoría económica tradicional y la teoría económica moderna. La primera está representada por el modelo de comercio internacional (Smith, 1776), y la segunda por el modelo de la ventaja competitiva de las naciones (Porter, 1991), también conocido como el "diamante de la ventaja nacional", del que se derivan los determinantes de la ventaja nacional competitiva, y de cuyo proceso de evolución se obtienen los dos estudios más reconocidos en el ámbito mundial que miden la competitividad de las naciones mediante la ejecución de sus modelos teóricos. Estos estudios se presentan en la subsección siguiente.

El proceso evolutivo del concepto de competitividad se muestra en el gráfico 1, donde se aprecia su desarrollo a través de los pensadores de los últimos tres siglos como un proceso de agregación que concluye en la propuesta actual de Michael Porter.

\section{Evolución del concepto de competitividad}

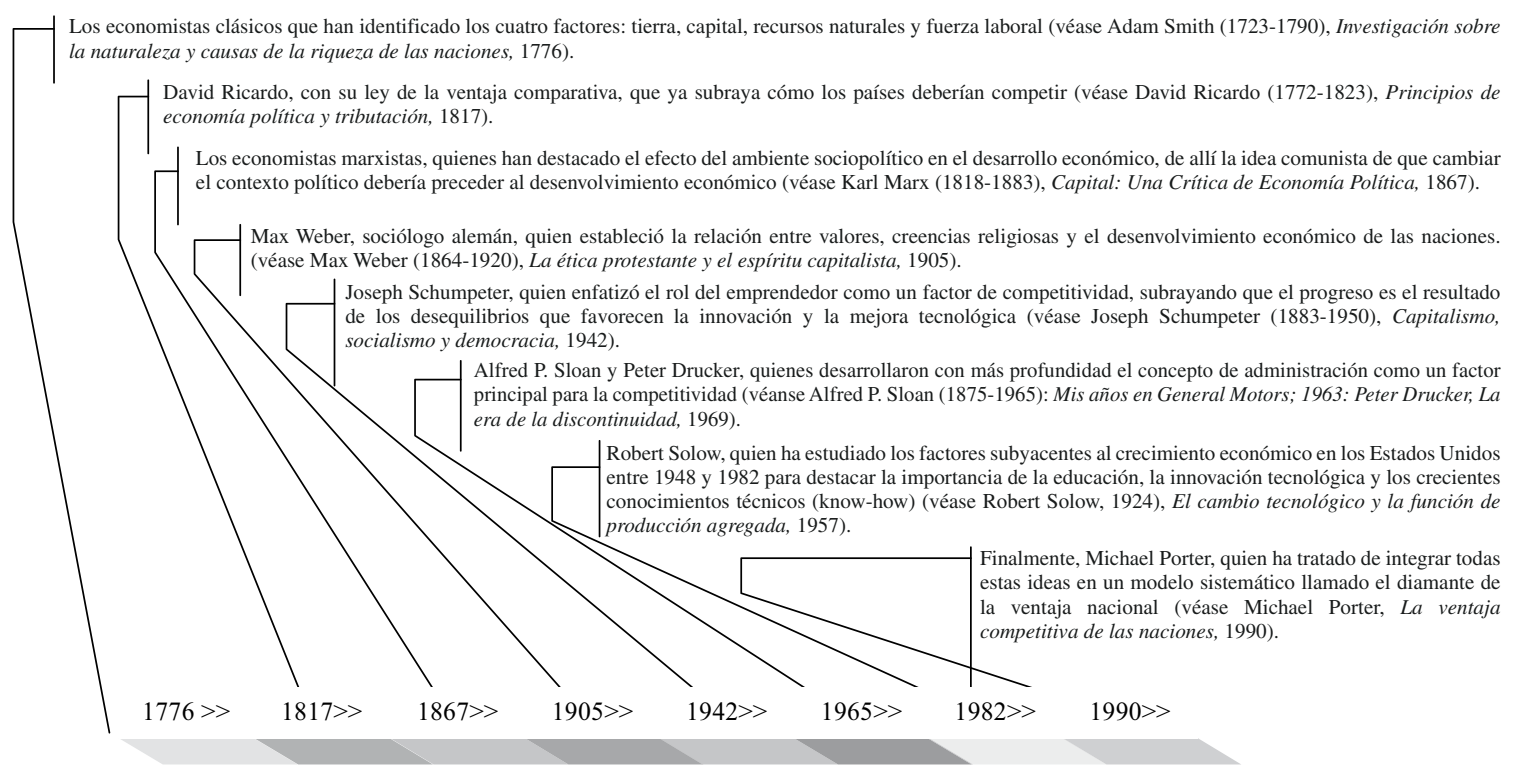

Fuente: S. Garelli, “The competitiveness of nations: the fundamentals”, IMD World Competitivenes Yearbook 2006, 2006 [en línea] http://www. IMD.ch/documents/wcc/content/Fundamentals.pdf 


\section{III}

\section{Índices de competitividad mundial y regional}

Los dos estudios más importantes a escala mundial que miden los determinantes de la competitividad de las naciones son el Informe de competitividad mundial y el Anuario de competitividad mundial, el primero desarrollado por el Foro Económico Mundial (WEF, por sus siglas en inglés, de aquí en adelante) y el segundo por el Instituto Internacional para el Desarrollo de la Capacidad de Gestión (IMD, por sus siglas en inglés, de aquí en adelante). Ambos se publican cada año sobre la base de información procedente de datos estadísticos y encuestas realizadas en cada país partícipe de la publicación. Instituciones asociadas se encargan de la recopilación de información y ejecución de la encuesta. En los dos estudios se utilizan factores de competitividad similares, obtenidos de la literatura y de análisis empíricos.

En el IMD se clasifican los datos en cuatro factores: i) desempeño económico, ii) eficiencia del gobierno, iii) eficiencia empresarial, iv) infraestructura; y cada uno de ellos se subdivide en cinco subfactores. En el WEF los datos se clasifican en 12 factores sin clasificaciones adicionales. Es importante resaltar que la clasificación del WEF corresponde a la edición 2008-2009 del Informe de competitividad mundial, debido a que en las tres ediciones precedentes se registraron importantes cambios en la metodología, que significaron variaciones en los factores con que se medía la competitividad.

El índice de competitividad global es liderado por el profesor Xavier Sala-i-Martin, asesor jefe de la Red de Competitividad Global del Wef (2008, pág. 3). Este índice se basa en 12 pilares de competitividad y ofrece un panorama detallado del escenario competitivo de los países del mundo en todos los niveles de desarrollo. Se elabora y publica anualmente desde 1979 y en su edición 2008-2009 se evaluaron 134 economías de países desarrollados y en vías de desarrollo.

La clasificación de las variables del Índice de Competitividad del Crecimiento (ICC) 2008-2009, según datos estadísticos o de la encuesta utilizada para medir la competitividad de las naciones evaluadas en este estudio, se compone de 110 variables, de las cuales $79(72 \%)$ provienen de la encuesta y la diferencia (31 variables) de información estadística de fuentes secundarias.

Por su parte, el Anuario de competitividad mundial se publica desde 1989 por el IMD con el fin de proporcionar a los líderes gubernamentales y empresariales a nivel mundial información sobre la situación y las principales tendencias en materia de competitividad. En 2008, el IMD publicó su índice respecto de 55 países de diferentes regiones del mundo, para lo cual cuenta con el apoyo de socios estratégicos en cada país involucrado.

El Anuario de competitividad mundial del IMD se basa en dos tipos de información:

i) indicadores estadísticos (datos concretos) recogidos especialmente de organismos internacionales como el Banco Mundial, la Organización de Cooperación y Desarrollo Económicos (OCDE), la Organización Mundial del Comercio (OMC), el Banco Interamericano de Desarrollo (BID) y las Naciones Unidas, entre otros, y

ii) encuestas anuales a empresarios a nivel mundial (datos abstractos).

Dos terceras partes del Anuario se basan en información estadística y una tercera parte en opiniones y percepciones del mundo empresarial, lo que lo diferencia del Índice de Competitividad del Crecimiento (ICC), donde se recogen mayoritariamente las opiniones empresariales. Otra diferencia con respecto al ICC es que mientras la teoría del WEF se basa en el modelo del diamante de Porter (1990), en el IMD se aplica una teoría propia, en la que se sostiene que los países gestionan sus entornos de acuerdo con cuatro fuerzas fundamentales que conforman el entorno competitivo.

En el cuadro 1 se aprecia un resumen con los índices y subíndices de: i) IMD 2008, ii) Heritage Foundation (2008) con su Índice de Libertad Económica, y iii) las últimas tres versiones del Informe de competitividad mundial del WEF. Para este último caso se destaca la evolución de los índices de Competitividad del Crecimiento y de Competitividad en los Negocios, que aparecieran en las publicaciones del WEF de años anteriores en el índice de competitividad global, que es la versión actual de este instituto suizo.

Los índices de competitividad regional implementados en América Latina se derivan principalmente de las investigaciones desarrolladas por Michael Porter y el Foro, al igual que del Anuario de competitividad mundial del IMD. Si bien es cierto que en varios de estos índices regionales no se logra continuidad en su elaboración, de todos ellos se concluye que su desarrollo fue esencial para apoyar los procesos de descentralización. Entre 


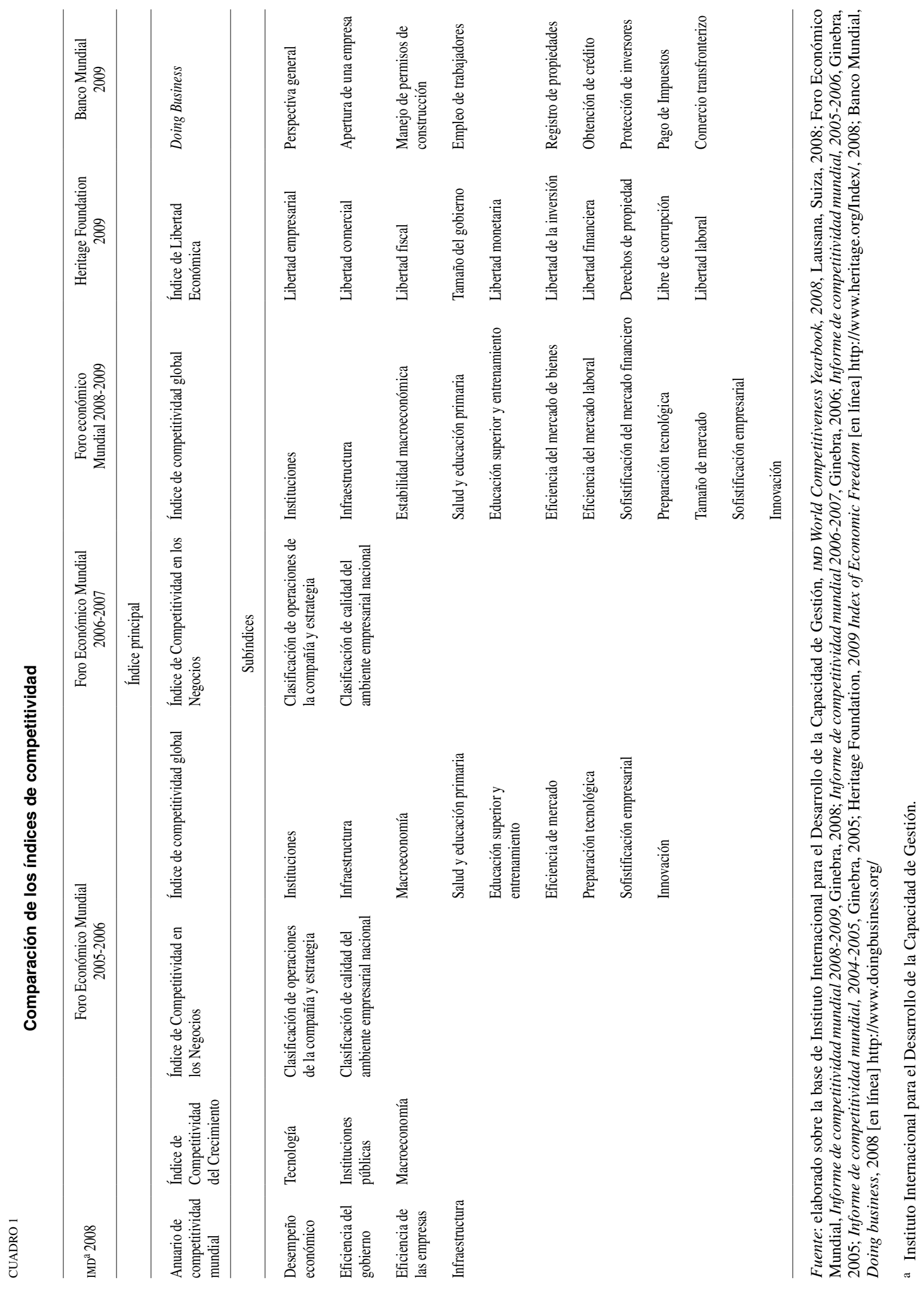




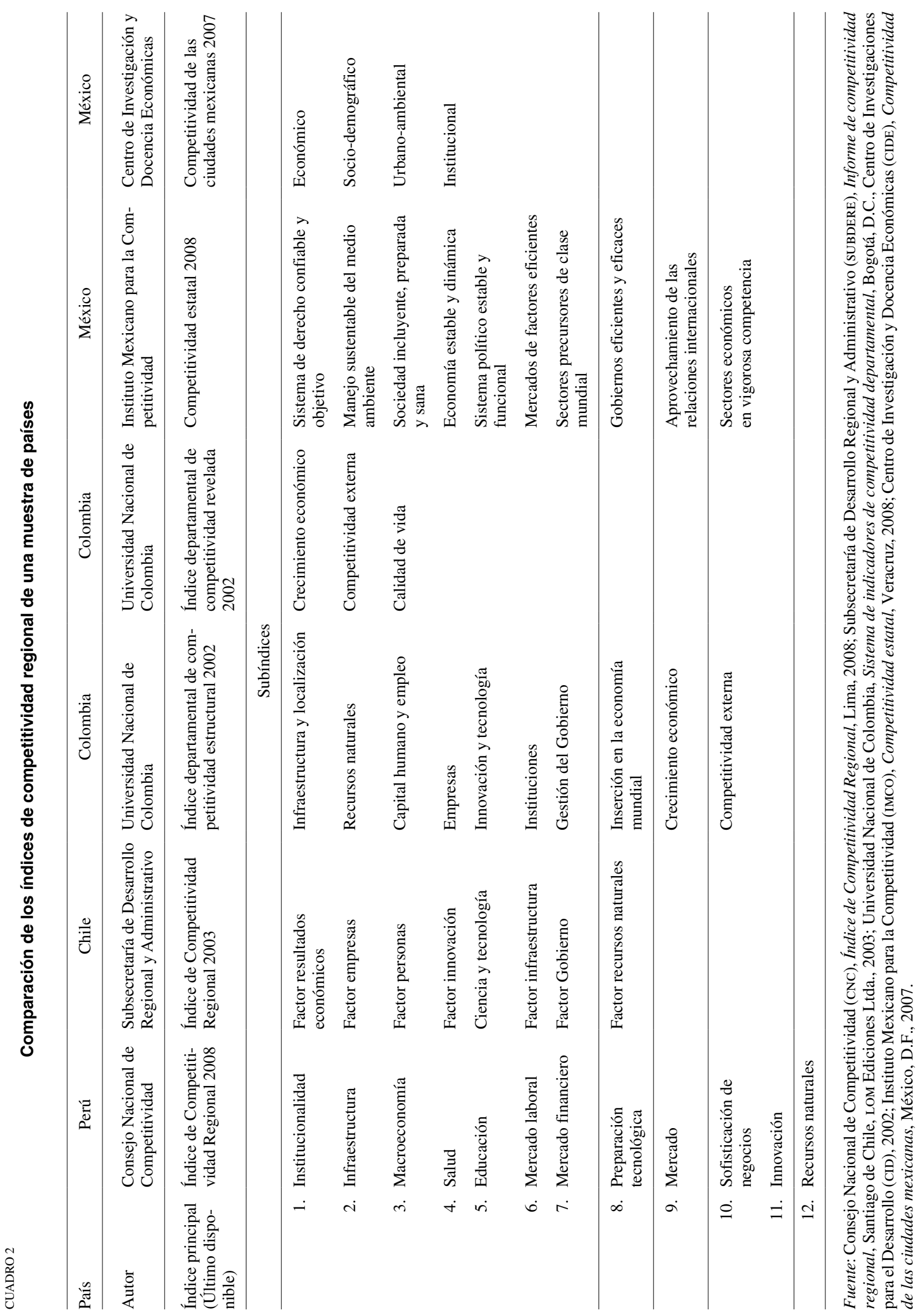


los índices latinoamericanos destacan los de México, Chile, Colombia y el Perú.

En el cuadro 2 se presenta una revisión de los determinantes de la competitividad regional según una muestra de índices de competitividad regional de América Latina; de ella se desprende que existen factores constantes independientemente del marco teórico que se haya utilizado.

\section{IV}

\section{Definición de competitividad regional}

En este contexto, la palabra regional hace referencia a la división geográfica de un país, que puede estar determinada por factores de diversa índole tales como demográficos, históricos, culturales, económicos y climáticos, entre otros.

Sobre la base del análisis de literatura previa, se deduce que la competitividad puede analizarse desde dos perspectivas. En primer lugar, como un conjunto de factores que determinan el nivel de productividad $y$, segundo, como un determinante del incremento sostenido del bienestar de las personas. A partir de estas perspectivas, la competitividad regional puede ser definida como la administración de recursos y capacidades para incrementar sostenidamente la productividad empresarial y el bienestar de la población de la región.

La definición propuesta se pone a prueba a través de la aplicación de las medidas de competitividad, que son aproximaciones estadísticas para evaluar la consistencia de la definición propuesta, contrastándola con evidencia empírica.

Esta evidencia se logra al comparar indicadores que reflejan el nivel de desarrollo de la economía de un país, como el producto interno bruto (PIB) per cápita, la productividad total de factores (PTF) o los índices similares al Índice de Competitividad Regional (ICR) de reconocido prestigio, como es el Índice de Desarrollo Humano (PNUD, 2006), y los de competitividad mundial del IMD y el WEF bajo determinados parámetros.
Este análisis se efectúa tomando como referencia el trabajo realizado por Tello (2004) en el informe sobre los factores de la competitividad en el Perú.

La primera medida de la competitividad tiene lugar entre el resultado final del Anuario de competitividad mundial del IMD y el PIB per cápita. Este análisis se realiza para las 55 economías que conformaron el Anuario del IMD en 2008. Del análisis se puede concluir que los países con una mejor posición en la clasificación de competitividad tienen un PIB per cápita más alto.

La segunda medida de la competitividad surge al comparar el Índice de Competitividad del Crecimiento del Foro Económico Mundial (2005a, último año en que fue presentado) y la tasa de crecimiento del PIB per cápita para el período 2003-2007. Del análisis se concluye que los países con un índice de competitividad del crecimiento más alto tuvieron un mayor crecimiento del PIB per cápita. El aumento de la competitividad, por lo tanto, se correlaciona con el crecimiento de la economía.

La tercera medida de la competitividad se verifica al relacionar el Índice de Competitividad del Crecimiento con la tasa de crecimiento promedio anual de la productividad total de los factores (PTF) para el período 2000-2004. Como resultado se observa una relación positiva, que indica que el incremento de la competitividad se relaciona con el crecimiento de la PTF. 


\section{V \\ Propuesta de un Índice de Competitividad Regional}

En la propuesta del Índice de Competitividad Regional (ICR) se considera un marco referencial para la construcción de índices, así como un modelo general y uno específico. Finalmente, se analiza y establece la forma de cálculo estadístico y se presentan todos sus componentes.

El ICR pertenece a la categoría de los indicadores sociales, generalmente vinculados a la investigación social y al diseño y gestión de proyectos sociales. Como indicador social, es una medida estadística de resumen, referida a la cantidad o magnitud de un conjunto de parámetros o atributos de una sociedad.

Tomando como referencia los indicadores que se suele utilizar para la gestión de proyectos, los indicadores sociales pueden clasificarse como simples o complejos (Bobadilla, Del Águila y Morgan, 1998). El ICR es un tipo de indicador social complejo debido a que requiere de un marco teórico y no cuenta con una forma simple de corroborar sus resultados.

Los indicadores también pueden clasificarse de acuerdo con su objeto de medición, pudiendo ser de impacto, de efecto y de cumplimiento (Bobadilla, Del Águila y Morgan, 1998). El ICR puede ser clasificado como un índice de impacto, porque mide la competitividad mediante los resultados obtenidos por una región al finalizar un tiempo determinado - un año por lo general- en el que las acciones de los agentes económicos han incrementado o reducido la competitividad.

En las investigaciones sociales se abordan fenómenos que difieren por su mayor o menor grado de complejidad y abstracción. El caso del ICR y del concepto de competitividad que lo soporta puede catalogarse como un concepto abstracto que empíricamente no es factible de observar y, por lo tanto, de medir. Para esto último se requiere realizar un proceso de descomposición y transformación denominado proceso de "operacionalización" (Lazarsfeld, 1958), que convierte la noción y el concepto de competitividad en un conjunto de indicadores que justamente permiten la observación empírica y, para el caso particular del presente estudio, la construcción de un índice.

Para Blalock (1970), en el proceso de "operacionalización" se debe considerar, primero, que la conceptualización deriva de reflexiones teóricas a partir de la revisión bibliográfica y de reflexiones propias y, segundo, que la medición permite asignar valores a los fenómenos sociales de acuerdo con determinadas reglas.

Para Lazarsfeld (1958), el proceso de "operacionalización" permite expresar los conceptos en términos de índices empíricos y se compone de las siguientes etapas: representación literaria del concepto; especificación del concepto, en que se identifican las dimensiones que son sus componentes o subdivisiones semánticas; y elección de indicadores para cada dimensión. Una vez elegidos los indicadores de las dimensiones, estos se sintetizan mediante la elaboración de índices.

En el gráfico 2 se observa el proceso de "operacionalización" del ICR, que es desarrollado a partir del esquema propuesto por Lazarsfeld (1958). Debido a que no existe concenso sobre una definición de competitividad, el proceso se inicia con la propuesta de la definición, la que se desagrega en sus componentes iniciales que son las dimensiones y que, además, dan forma a los pilares que componen la definición de competitividad (en este caso, competitividad regional). Finalmente, se ponderan las variables de los factores contenidos en los pilares y se determinan las combinaciones posibles. Ponderar implica asignar pesos en un intento de expresar diferencias en la importancia relativa en el ICR.

La metodología propuesta deriva tanto de la revisión de experiencias similares en la construcción de índices de competitividad como de la revisión bibliográfica. Los factores utilizados, las técnicas estadísticas aplicadas y la información requerida permiten que el ICR pueda ser generalizado y replicado en otros países que cuenten con un índice de desarrollo humano similar (PNUD, 2006), a fin de minimizar la repercusión de las diferencias económicas y sociales inherentes a cada país. Las adaptaciones necesarias para la aplicación del ICR en países no deberían invalidar la comparación entre sus regiones. Esto con el afán de contar con una base que permita ampliar el espectro de comparación y disponer de referentes cada vez más avanzados que representen objetivos nacionales por alcanzar. 
GRÁFICO 2

\section{Proceso de "operacionalización” del Índice de Competitividad Regional (ICR)}

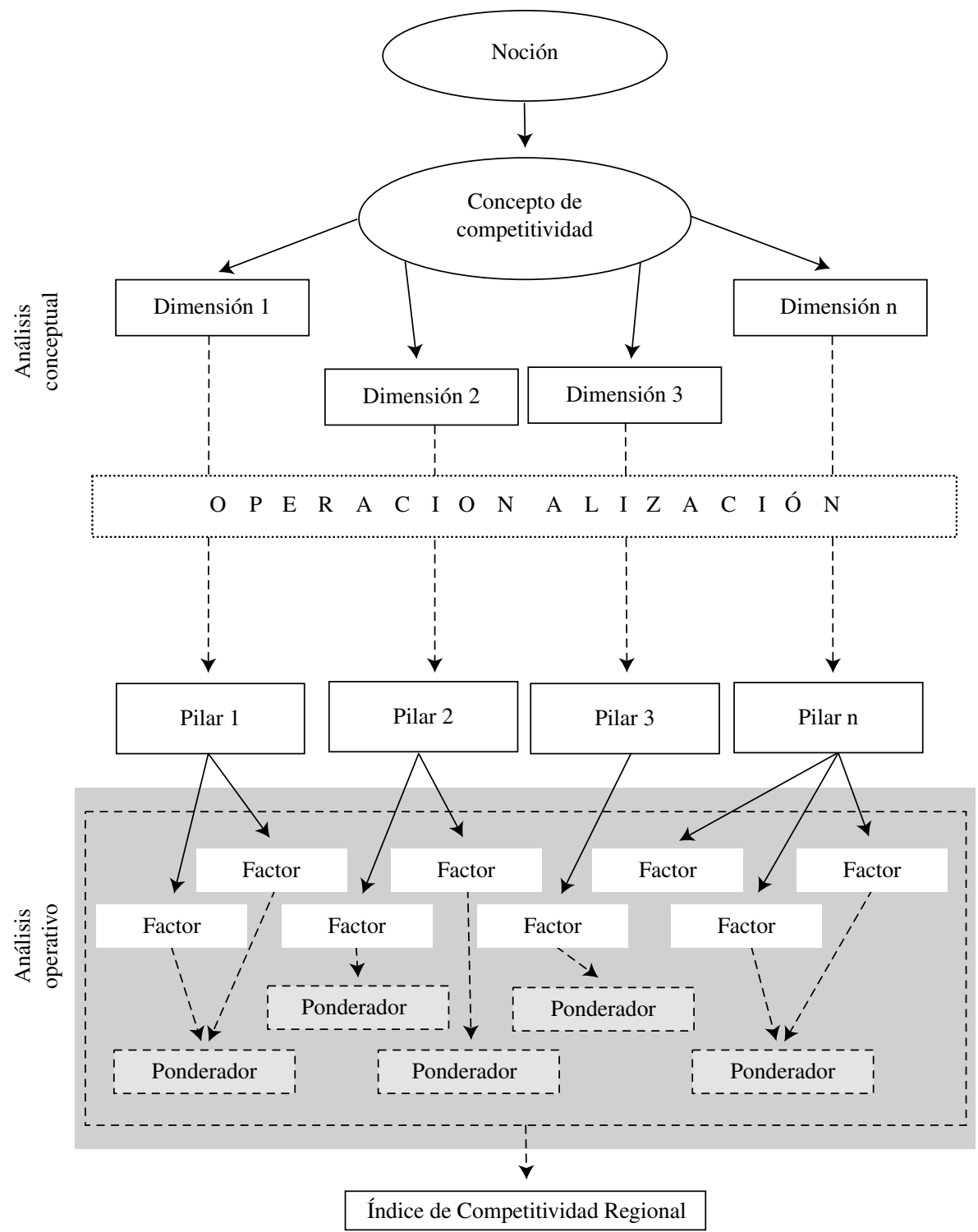

Fuente: P.F. Lazarsfeld, "Evidence and inference in social research", American Academy of Arts \& Sciences, vol. 87, № 4, Cambridge, Massachusetts, American Academy of Arts \& Sciences. 


\section{VI}

\section{Determinantes de la competitividad regional}

Los determinantes de la competitividad regional, que para efectos de este estudio se denominarán pilares, han sido establecidos sobre la base de la literatura previa y el análisis de las experiencias internacionales. No existe un conjunto de determinantes de la competitividad que se derive de un consenso explícito; por el contrario, la identificación de pilares de la competitividad se comporta, en la práctica, como un proceso de elección, que responde a distintos criterios. Los criterios pueden referirse a disponibilidad, frecuencia y concordancia con el concepto (Joy Way, 2004); o bien a i) consistencia con la definición y marco conceptual, ii) soporte estadístico, en el sentido que el factor esté relacionado estadísticamente con los indicadores de desempeño de una economía, iii) que el factor pueda ser sujeto de alguna medida (cualitativa o cuantitativa) y ser fácilmente identificable respecto del resto de los factores (Tello, 2004).

Este proceso de elección de pilares es un común denominador de los índices de competitividad, especialmente de los índices regionales. En el proceso se aplican diferentes metodologías, desde el desarrollo de talleres o entrevistas con expertos hasta la aplicación de modelos propios. Para el proceso de identificación de los pilares de la competitividad regional propuestos se han tomado como modelo de referencia las bases de las ventajas competitivas regionales de Kitson, Martin y Tyler (2004) (véase el gráfico 3). La idea de competitividad regional vinculada a estas bases captura la noción de que - a pesar del hecho de que hay firmas competitivas y no competitivas en cada región- existen elementos comunes en una región que afectan a la competitividad de todas las empresas. El enfoque que plantean los autores es el de externalidades regionales, es decir, aquellos recursos que están fuera de la empresa pero que se utilizan directa o indirectamente y repercuten en su eficiencia, innovación, flexibilidad y dinamismo; en suma, en su productividad y ventaja competitiva.

El proceso seguido en el presente estudio para determinar los pilares de la competitividad regional a partir de las bases de las ventajas competitivas regionales (Kitson, Martin y Tyler, 2004) comprende dos etapas: la generalización de las definiciones de

GRÁFICO 3

Bases de las ventajas competitivas regionales

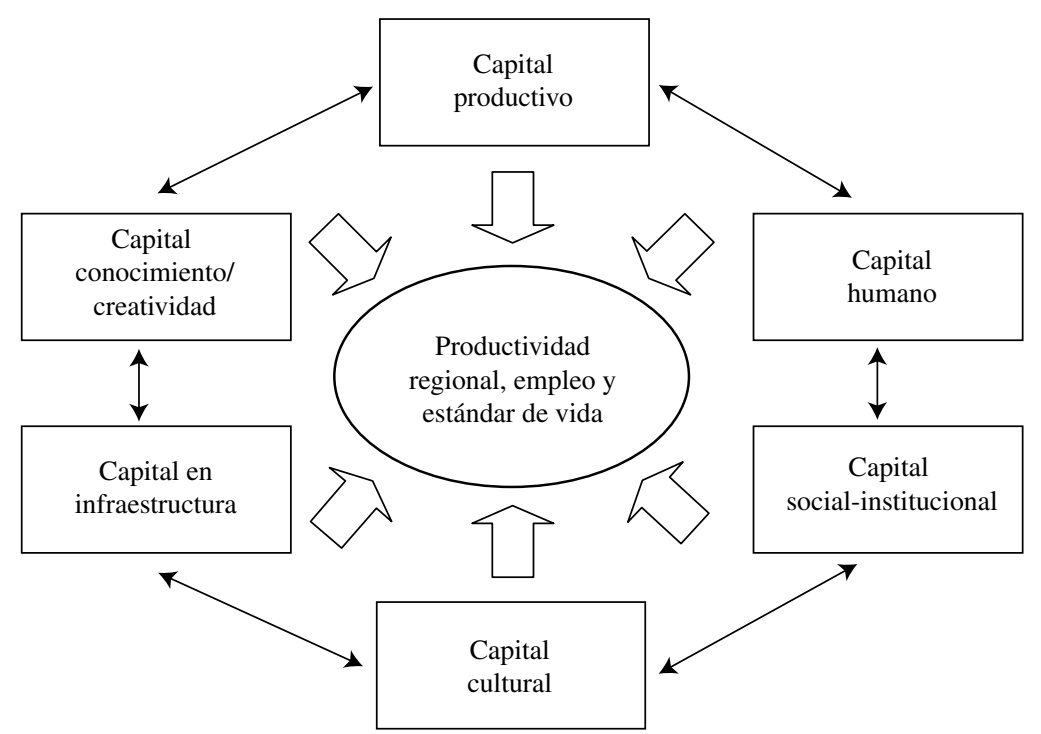

Fuente: M. Kitson, R. Martin y P. Tyler, “Regional competitiveness: an elusive yet key concept?”, Regional Studies, vol. 38, N 9, Londres, Taylor \& Francis, 2004. 
las seis bases (véase el cuadro 3) y el análisis de su aplicabilidad, contrastando las bases con los índices de competitividad mundial y regional existentes (véase el cuadro 4).

Los pilares se derivan principalmente de las bases de las ventajas competitivas regionales (Kitson, Martin y Tyler, 2004), con excepción del capital cultural, debido a que no se ha encontrado evidencia empírica acorde con la definición de dichas bases en las experiencias internacionales relacionadas con la inclusión del capital cultural. Asimismo, se observa que ni en los índices mundiales ni en los índices regionales se considera al capital cultural como un factor individual o variable. En varios de ellos, al igual que en los pilares del presente estudio, se lo considera vinculado a la educación y por ende al capital humano.

A continuación se describen los pilares de la competitividad regional propuestos y sus fundamentos.

\section{Gobierno e instituciones (P1)}

El primer pilar de la competitividad regional es el que compete al gobierno y sus instituciones. Con respecto al gobierno, es importante anotar cuáles son sus principales funciones a fin de poder sentar las bases de los componentes con respecto a su competitividad. Si bien es cierto que gobierno no es lo mismo que Estado, se puede entender que un gobierno ejerce el poder del Estado, es decir, ejerce actividades estatales denominadas comúnmente poderes del Estado. Por lo tanto, el papel estatal se puede analizar en tres frentes: el microeconómico, el macroeconómico y el institucional (León, 2003).

El pilar gobierno e instituciones surge del frente microeconómico del papel del Estado; es decir — según la teoría microeconómica-, en competencia perfecta el libre mercado permite alcanzar el equilibrio. Sin embargo, este escenario es utópico debido a que existen fallas o distorsiones en el mercado que evitan que este opere en tal situación de eficiencia. La más común de estas fallas es la existencia de bienes públicos que necesitan ser administrados con criterios económicos y sociales, considerando las externalidades o generando monopolios y oligopolios. Es en estos casos que surge el fundamento teórico para la intervención del Estado y, por ende, el fundamento para el accionar de los gobiernos a través de sus instituciones, que tiene sus raíces en Keynes (1936), quien vislumbraba el papel del Estado.

\section{Desarrollo económico (P2)}

Al segundo pilar de la competitividad regional le compete, primero, el desempeño de la economía de la región, segundo, el proceso de internacionalización y, tercero, la capacidad de generación de empleo. El pilar desarrollo económico se vincula a la teoría del crecimiento económico cuyo modelo más representativo en la economía

CUADRO 3

\section{Clasificación de los pilares de los índices de competitividad mundial de acuerdo con las bases de la ventaja competitiva regional}

\begin{tabular}{|c|c|c|}
\hline $\begin{array}{l}\text { Bases de la ventaja } \\
\text { competitiva regional }\end{array}$ & $\begin{array}{l}\text { Anuario de competitividad mundial } \\
2008\end{array}$ & $\begin{array}{l}\text { Índice de competitividad global } \\
2008-2009\end{array}$ \\
\hline 1. Capital productivo & - Desarrollo económico & $\begin{array}{l}\text { - Estabilidad macroeconómica } \\
\text { - } \quad \text { Sofisticación del mercado financiero } \\
\text { - Tamaño de mercado }\end{array}$ \\
\hline 2. Capital humano & - Desarrollo económico & - Eficiencia del mercado laboral \\
\hline 3. Capital cultural & & \\
\hline 4. Capital social-institucional & - Eficiencia del gobierno & - Instituciones \\
\hline 5. Capital en infraestructura & - Infraestructura & $\begin{array}{l}\text { - Infraestructura } \\
\text { - } \text { Salud y educación primaria } \\
\text { - } \text { Educación superior y entrenamiento }\end{array}$ \\
\hline 6. Capital conocimiento / creatividad & - Eficiencia de las empresas & $\begin{array}{l}\text { - Innovación } \\
\text { - Eficiencia del mercado de bienes } \\
\text { - Preparación tecnológica } \\
\text { - } \quad \text { Sofisticación empresarial }\end{array}$ \\
\hline
\end{tabular}

Fuente: elaborado sobre la base de M. Kitson, R. Martin y P. Tyler, "Regional competitiveness: an elusive yet key concept?", Regional Studies, vol. 38, $\mathrm{N}^{\circ}$ 9, Londres, Taylor \& Francis, 2004; Instituto Internacional para el Desarrollo de la Capacidad de Gestión, IMD World Competitiveness Yearbook, 2008, Lausana, Suiza, 2008; Foro Económico Mundial, Índice de competitividad mundial, Ginebra, 2008. 
CUADRO 4 Clasificación de los factores de los índices de competitividad regional
de acuerdo con las bases de la ventaja competitiva regional

\begin{tabular}{|c|c|c|c|c|}
\hline $\begin{array}{l}\text { Bases de la ventaja } \\
\text { competitiva regional }\end{array}$ & $\begin{array}{c}\text { Índice de } \\
\text { Competitividad } \\
\text { Regional } 2003 \text { de Chile }\end{array}$ & $\begin{array}{l}\text { Índice departamental de } \\
\text { competitividad estructural } \\
2002 \text { de Colombia }\end{array}$ & $\begin{array}{c}\text { Competitividad estatal } 2008 \\
\text { de México }\end{array}$ & $\begin{array}{l}\text { Competitividad } \\
\text { de las ciudades } \\
\text { mexicanas } 2007\end{array}$ \\
\hline \multirow[t]{4}{*}{ 1. Capital productivo } & $\begin{array}{l}\text { - Factor resultados } \\
\text { económicos }\end{array}$ & - Inserción en la economía mundial & - Economía estable y dinámica & - Económico \\
\hline & $\begin{array}{l}\text { - Factor recursos } \\
\text { naturales }\end{array}$ & - Crecimiento económico & $\begin{array}{l}\text { - Sectores económicos en vigorosa } \\
\text { competencia }\end{array}$ & \\
\hline & & - Competitividad externa & $\begin{array}{l}\text { - Aprovechamiento de las relaciones } \\
\text { internacionales }\end{array}$ & \\
\hline & & - Recursos naturales & $\begin{array}{l}\text { - Mercados de factores eficientes } \\
\text { - Sectores precursores de clase mundial } \\
\text { - Manejo sostenible del medio ambiente }\end{array}$ & \\
\hline 2. Capital humano & - Factor personas & - Capital humano y empleo & & \\
\hline 3. Capital cultural & & & & \\
\hline 4. Capital social-institucional & - Factor gobierno & $\begin{array}{l}\text { - Instituciones } \\
\text { - Gestión del gobierno }\end{array}$ & $\begin{array}{l}\text { - Sistema político estable y funcional } \\
\text { - Gobiernos eficientes y eficaces } \\
\text { - Sistema de derecho confiable y objetivo } \\
\text { - Sociedad incluyente, preparada y sana }\end{array}$ & - Institucional \\
\hline 5. Capital en infraestructura & - Factor infraestructura & - Infraestructura y localización & & $\begin{array}{l}\text { - Urbano-ambiental } \\
\text { - Sociodemográfico }\end{array}$ \\
\hline $\begin{array}{l}\text { 6. Capital conocimiento/ } \\
\text { creatividad }\end{array}$ & $\begin{array}{l}\text { - Factor empresas } \\
\text { - Factor innovación } \\
\text { - Ciencia y tecnología }\end{array}$ & $\begin{array}{l}\text { - Empresas } \\
\text { - Innovación y tecnología }\end{array}$ & & \\
\hline
\end{tabular}

Fuente: elaborado sobre la base de M. Kitson, R. Martin y P. Tyler, “Regional competitiveness: an elusive yet key concept?", Regional Studies, vol. 38, N 9, Londres, Taylor \& Francis, 2004; ; Subsecretaría de Desarrollo Regional y Administrativo (subDERE), Informe de competitividad regional, Santiago de Chile, LOM Ediciones Ltda., 2003; Universidad Nacional de Colombia, Sistema de indicadores de competitividad departamental, Bogotá, D.C., Centro de Investigaciones para el Desarrollo (CID), 2002; Instituto Mexicano para la Competitividad (IMCO), Competitividad estatal, Veracruz, 2008; Centro de Investigación y Docencia Económicas (CIDE), Competitividad de las ciudades mexicanas, México, D.F., 2007.

moderna de crecimiento es el de Solow (1956), quien atribuye el crecimiento a la acumulación de capital, el incremento de la fuerza de trabajo y el cambio tecnológico. Sin embargo, a diferencia del esquema contable de Solow (1956), y siguiendo el objetivo de describirlo, el propósito del pilar desarrollo económico es medir el efecto del crecimiento económico, el que además tiene que responder a criterios de sostenibilidad, responsabilidad social y cuidado medioambiental.

Para Sachs y Larraín (1994), el crecimiento económico es necesario para mejorar el nivel de vida de una población que va en aumento. Por su parte, Malthus (1798) argumentaba que el incremento de la población estaría limitado por la cantidad de recursos que puede proveer la Tierra, sosteniendo que el crecimiento de la población supera las posibilidades del planeta (recursos) de proveer subsistencia al hombre, y que inevitablemente la muerte, bajo la forma de guerras o gigantescas hambrunas, equilibraría la relación.

\section{Infraestructura productiva (P3)}

La infraestructura es la intervención primaria del ser humano en el territorio para acceder a él y activar su potencial de desarrollo (CAF, 2008). El nivel de la infraestructura de una región se vincula estrechamente con su grado de desarrollo y constituye, en caso de retraso, una severa restricción de la posibilidad de alcanzar grandes avances en el bienestar material de su población.

Uno de los índices especializados más importantes es el ranking de infraestructura que publica América Economía (2008). El pilar infraestructura productiva recoge la metodología del mencionado ranking y se plantea como la capacidad actual de cada región para 
sustentar la productividad y competitividad de los negocios. En ese sentido, se puede afirmar que los efectos de la infraestructura se aprecian en la creación de empleo y en el mejoramiento de la competitividad de la región y de la calidad de vida de sus ciudadanos.

\section{Capital humano (P4)}

El capital humano es el valor del potencial de obtención de ingreso que poseen los individuos. A pesar de tener un componente de recurso natural, procede en su mayor parte de inversiones en educación, adiestramiento y salud. Estas inversiones permiten que el capital humano tenga mayor productividad (Larroulet y Mochon, 1995).

La ciencia económica empieza a valorar el papel del capital humano a partir de los aportes de Schultz (1961), quien planteó por primera vez que las habilidades y conocimientos son una forma de capital. Pocos años después, Becker (1964) considera al capital humano como un factor económico primario en su estudio sobre el conocimiento. Sin embargo, el aporte más significativo, el de introducir las habilidades humanas en la función de producción, proviene de los trabajos de Uzawa (1965) y Lucas (1988). De acuerdo con estos autores, la función de producción de una economía podría representarse mediante una función de Cobb-Douglas ( $\left.Y=A \cdot K^{\alpha} \cdot H^{1-\alpha}\right)$, en que se considera que la producción (Y) está determinada, además del capital físico $(\mathrm{K})$, por el capital humano $(\mathrm{H})$.

\section{Eficiencia de las empresas (P5)}

La prosperidad nacional se crea, no se hereda, y depende de la capacidad de su industria para innovar y mejorar (Porter, 2001). Es decir, la competitividad es sinónimo de productividad y esta se logra fomentando la innovación, la que se ve impulsada por los cuatro atributos de una nación, la que a su vez depende de la capacidad de sus empresas para innovar y mejorar.

A pesar de que el sustento del enfoque con respecto a la industria, las empresas y la productividad es una base sólida para el pilar eficiencia de las empresas, existe el enfoque de competitividad sistémica (Esser y otros, 1996, págs. 39-52) que, sin oponerse al enfoque de Porter (2001), refuerza la visión con respecto al papel de la empresa. El enfoque sistémico, al nivel micro, se vincula directamente con el desarrollo de la empresa y de su entorno inmediato. En este enfoque se postula que las empresas, para poder afrontar con éxito las nuevas exigencias, necesitan reorganizarse tanto a nivel interno como dentro del entorno que las rodea.

\section{VII}

\section{Factores y variables de la competitividad regional}

Los factores corresponden a los elementos que componen los pilares. En conjunto, le otorgan al pilar un determinado significado, derivado de las variables inmersas y de las ponderaciones que reciben. La determinación de los factores y variables es el resultado de un proceso propio de cada país, que se establece por el cumplimiento de criterios que es necesario seguir para lograr una selección acorde con las características del país. Los criterios para la determinación de los factores y variables del Índice de Competitividad Regional son:

- Que las variables que componen los factores sean recopiladas de una fuente oficial. Existe un primer grupo de instituciones que generan información, ya sea obteniéndola mediante trabajo de campo o realizando cálculos de escritorio, y un segundo grupo que recopila información del primer grupo para compendiarla y generar publicaciones estadísticas.
- Que tenga un registro histórico de más de tres años continuos. Este criterio garantiza la continuidad de la información y que no se trate de una variable que se calculó atendiendo a una necesidad en particular; por lo tanto, se reduce la probabilidad de no contar con ella en el año siguiente.

- Que tenga desagregación por regiones.

- Que la metodología que se utiliza en las fuentes sea rigurosa y estable en el tiempo con el fin de no afectar al potencial de análisis comparativo.

Estos criterios se aplican en el proceso de selección de factores y variables en las fuentes oficiales de información estadística del país y se consideran en el marco que constituyen los pilares identificados.

El pilar "gobierno e instituciones" se compone de factores que cuantifican la capacidad de los gobiernos regionales y locales para cumplir su rol de Estado que 
provee servicios a sus habitantes (recursos, gasto, inversión, seguridad, presencia del Estado) y alentar así el desarrollo sostenido de su región.

Para el pilar "desarrollo económico" se considera entre los factores el crecimiento de la producción y el empleo, entre otros, que no solo son el resultado del último año, sino del último período o ciclo económico representativo. De igual manera, las variables no solo son corrientes (valores actuales), sino también reales (valores deflactados) a fin de evitar el sesgo que implica la volatilidad de los precios. Por su parte, en el comercio exterior no solo se considera el valor exportado, sino también el volumen y, en mayor grado, el proceso de internacionalización y diversificación de la región.

Con respecto a los factores que componen el pilar "infraestructura productiva", en ellos se toma como referencia el concepto de base física (Joy Way, 2004), que implica el conjunto de factores físicos sobre los que descansa la competitividad de una región. Por lo tanto, se incluye el soporte de infraestructura — como la red vial, energía y transporte- y la forma particular de ordenamiento de los factores de la producción de cada región, como la conectividad y el turismo.

En el pilar "capital humano" se consideran como factores aquellos que guardan directa relación con las teorías presentadas. Se tienen en cuenta la educación escolar y la superior, pero distinguiéndolas entre privada y pública; también se considera la formación laboral distinta a la universitaria, y finalmente la salud.

Por último, en el pilar "eficiencia de las empresas" se integran tanto los factores directamente vinculados a la empresa como la productividad, habilidades empresariales e innovación, como los de su entorno inmediato, tales como el clima de negocios y la generación de empleo.

Las variables constituyen la definición general del indicador utilizado y juntas conforman el factor. Cada una de las variables se vincula a un indicador, ya sea un indicador simple o relativo. Un indicador simple es el que muestra el valor absoluto de la variable, y uno relativo el que se calcula con respecto a alguna otra variable, como la población, el PIB, entre otros. En los cuadros 5 al 9 se muestran los factores y las variables clasificadas por cada factor para cada uno de los pilares.

CUADRO 5

\section{Componentes del pilar gobierno e instituciones}

\begin{tabular}{ll}
\hline Factor & Variable de medición \\
\hline 1. Recursos recaudados & $\begin{array}{l}\text { Recaudación directa relativa } \\
\text { Recaudación directa }\end{array}$ \\
2. Recursos transferidos & $\begin{array}{l}\text { Ingresos por transferencias relativos } \\
\text { Ingresos por transferencias }\end{array}$ \\
3. Gasto en inversión & $\begin{array}{l}\text { Participación del gasto en inversión } \\
\text { Gasto en inversión }\end{array}$ \\
4. Seguridad pública & $\begin{array}{l}\text { Delitos } \\
\text { Faltas } \\
\text { Terrorismo } \\
\text { 5. Presencia del Estado }\end{array}$ \\
& $\begin{array}{l}\text { Presencia de escuelas primarias y } \\
\text { secundarias } \\
\text { Presencia de establecimientos de salud } \\
\text { Presencia de una comisaría o puesto } \\
\text { policial } \\
\text { Presencia de la municipalidad }\end{array}$ \\
\hline
\end{tabular}

Fuente: elaboración propia. 
CUADRO 6

Componentes del pilar desarrollo económico

\begin{tabular}{ll}
\hline Factor & Variable de medición \\
\hline 1. Tamaño económico & $\begin{array}{l}\text { PIB real } \\
\text { PIB per cápita }\end{array}$ \\
2. Crecimiento económico & Crecimiento del PIB real y corriente \\
3. Internacionalización & $\begin{array}{l}\text { Exportaciones en valor } \\
\text { Exportaciones como porcentaje del } \\
\text { PIB y volumen } \\
\text { Dinámica exportadora }\end{array}$ \\
& $\begin{array}{l}\text { Países de destino } \\
\text { Productos }\end{array}$ \\
4. Diversificación & PEA ocupada \\
& PEA ocupada relativa \\
Remuneración de ejecutivos, \\
Empleo
\end{tabular}

Fuente: elaboración propia.

PEA: Población económicamente activa.

CUADRO 7

\section{Componentes del pilar infraestructura productiva}

Factor Variable de medición

\begin{tabular}{ll}
\hline 1. Energía & Energía eléctrica \\
& Clientes libres y consumo de clientes libres \\
& Clientes regulados y consumo de clientes \\
& regulados
\end{tabular}

2. Red vial Red vial nacional y densidad de red nacional Red vial departamental y densidad de red departamental

Red vial vecinal y densidad

3. Transporte Transporte terrestre y densidad del transporte terrestre

Transporte aéreo y densidad del transporte aéreo

Tráfico de carga internacional en aeropuertos Tráfico de carga de exportación en puertos

4. Turismo Hoteles de $1,2,3,4$ y 5 estrellas

Albergues

Otros establecimientos

5. Conectividad Telefonía fija y densidad telefonía fija Telefonía celular y densidad telefonía celular

Fuente: elaboración propia.
CUADRO 8

\section{Componentes del pilar capital humano}

\begin{tabular}{|c|c|}
\hline Factor & Variable de medición \\
\hline $\begin{array}{l}\text { 1. Educación } \\
\text { escolar }\end{array}$ & $\begin{array}{l}\text { Comprensión de lectura y matemáticas en la } \\
\text { primaria } \\
\text { Comprensión de lectura y matemáticas en la } \\
\text { secundaria }\end{array}$ \\
\hline $\begin{array}{l}\text { 2. Educación } \\
\text { superior pública }\end{array}$ & $\begin{array}{l}\text { Graduados de universidad pública y densidad } \\
\text { Titulados de universidad privada y densidad }\end{array}$ \\
\hline $\begin{array}{l}\text { 3. Educación } \\
\text { superior privada }\end{array}$ & $\begin{array}{l}\text { Graduados de universidad privada y densidad } \\
\text { Titulados de universidad privada y densidad }\end{array}$ \\
\hline $\begin{array}{l}\text { 4. Educación } \\
\text { superior privada }\end{array}$ & $\begin{array}{l}\text { Densidad de graduados de universidad } \\
\text { privada }\end{array}$ \\
\hline $\begin{array}{l}\text { 5. Formación } \\
\text { laboral }\end{array}$ & $\begin{array}{l}\text { Graduados de universidad privada } \\
\text { Densidad de titulados de universidad privada } \\
\text { Titulados de universidad privada } \\
\text { Densidad de centros de formación tecnológica }\end{array}$ \\
\hline 6. Salud & $\begin{array}{l}\text { Centros de formación tecnológica } \\
\text { Centros de formación ocupacional y densidad } \\
\text { Mortalidad infantil } \\
\text { Expectativa de vida } \\
\text { Morbilidad } \\
\text { Cobertura médica }\end{array}$ \\
\hline
\end{tabular}

Fuente: elaboración propia.

CUADRO 9

\section{Componentes del pilar eficiencia de las empresas}

\begin{tabular}{|c|c|}
\hline Factor & Variable de medición \\
\hline 1. Productividad & $\begin{array}{l}\text { Productividad media del trabajo (PMT) } \\
\text { Variación de la productividad media del trabajo } \\
\text { PEA ocupada }\end{array}$ \\
\hline $\begin{array}{l}\text { 2. Clima de } \\
\text { negocios }\end{array}$ & $\begin{array}{l}\text { Número de empresas } \\
\text { Penetración y cobertura del sistema financiero } \\
\text { Nacimiento de empresas } \\
\text { Esfuerzo para desarrollar empresas } \\
\text { Presencia de empresas exitosas }\end{array}$ \\
\hline $\begin{array}{l}\text { 3. Habilidades } \\
\text { empresariales }\end{array}$ & $\begin{array}{l}\text { Capacidad gerencial } \\
\text { Visión de largo plazo } \\
\text { Capacidad de adaptación e internacionalización }\end{array}$ \\
\hline 4. Innovación & $\begin{array}{l}\text { Existencia de productos / servicios innovadores } \\
\text { Casos de empresas o personas innovadoras } \\
\text { Creación de nuevos productos o servicios } \\
\text { Mejora de técnicas y procesos }\end{array}$ \\
\hline 5. Innovación & Existencia de productos / servicios innovadores \\
\hline $\begin{array}{l}\text { 6. Generación } \\
\text { de empleo }\end{array}$ & $\begin{array}{l}\text { Casos de empresas o personas innovadoras } \\
\text { Creación de nuevos productos o servicios } \\
\text { Mejora de técnicas y procesos } \\
\text { Acceso a puestos bien remunerados } \\
\text { Oportunidades para independientes } \\
\text { Empleo estable } \\
\text { Nivel salarial } \\
\text { Sobrecosto laboral }\end{array}$ \\
\hline
\end{tabular}

Fuente: elaboración propia.

PEA: Población económicamente activa. 


\section{VIII}

\section{Cálculo del Índice de Competitividad Regional}

El Índice de Competitividad Regional (ICR) se puede representar mediante la siguiente notación:

$$
I C R=\frac{\sum_{k=1}^{l} \text { Pilar }_{k}}{l}
$$

donde el ICR es el promedio de los $l$ pilares que lo componen y donde cada pilar $\left(\right.$ Pilar $_{k}$ ) está representado por el promedio de los $m$ factores que lo componen.

$$
\text { Pilar }_{k}=\frac{\sum_{k=1}^{m} F_{j}}{m}
$$

Finalmente, el factor $\left(F_{j}\right)$ es la sumatoria de las $n$ variables que lo componen ponderado por

$$
F_{j} \sum_{i=1}^{n} V_{i} P_{i}
$$

es decir, un factor $\left(P_{i}\right)$

donde $V_{i}=$ Variable $i$

y $P_{i}=$ ponderación de la variable $i$

En el cálculo del ICR se enfrentan dos tipos de problemas comunes a todo esfuerzo de construcción de índices: i) ¿cómo "uniformizar" criterios diferentes?, y ii) ¿cómo integrar los criterios "uniformizados" en el índice? Ambos problemas pueden sintetizarse en una sola pregunta: ¿cómo transformar las variables para que puedan ser integradas en un solo índice?
Con respecto al primer problema, existen tres opciones que son las más aceptadas en la estadística para la "uniformización" de criterios y que derivan de variables o indicadores disímiles: i) conversión de escala, ii) rango percentil, y iii) resultado estándar. En el cuadro 10 se presenta un resumen de las capacidades de cada método con respecto a los criterios de análisis descritos.

Asimismo, se desprende que todos los métodos no cumplen, a lo menos, con uno de los criterios establecidos. El primer criterio puede ser el más subjetivo de los tres y la observación negativa para el resultado estándar se debe a la comparación relativa con los otros métodos que resultan ser más simples de entender porque no presentan valores negativos y una escala acotada.

La dificultad relativa de interpretación del resultado estándar puede superarse aplicando la conversión de escala, luego de calculado el resultado estándar. Además, el resultado estándar es el único método que cumple con el tercer criterio de poder brindar una referencia objetiva de la distancia relativa entre los resultados de cada región. Por las razones expuestas, el método del resultado estándar complementado con la conversión de escala, al que llamaremos simplemente estandarización, es el mejor método para calcular el ICR.

No es factible establecer un criterio uniforme para asignar las ponderaciones a cada una de las variables que componen el ICR; sin embargo, se pueden establecer algunos principios a fin de reducir la arbitrariedad. Estos principios tienen que ver fundamentalmente con la unidad de medida de las variables, dándose los dos siguientes casos:

\begin{tabular}{lccc}
\hline Método de "uniformización" & $\begin{array}{c}\text { ¿Brinda facilidad } \\
\text { de interpretación? }\end{array}$ & $\begin{array}{c}\text { ¿Permite ordenar } \\
\text { jerárquicamente? }\end{array}$ & $\begin{array}{l}\text { ¿Permite calcular las } \\
\text { distancias relativas? }\end{array}$ \\
\hline Conversión de escala & Sí & Sí & No \\
Rango percentil & Sí & Sí & No \\
Resultado estándar & No & Sí & Sí \\
\hline
\end{tabular}

Fuente: elaboración propia. 
i) Variables con unidad de medida original, es decir, la unidad derivada de la fuente principal de la que fue recopilada.

ii) Variables con unidad de medida transformada, es decir, la unidad derivada de un proceso simple de relativización de la variable con respecto a una variable que dimensione algún aspecto de trascendencia para la región, denominado variable transformadora.

El primer criterio en la asignación de ponderaciones de cada una de las variables que componen cada pilar del ICR es que se consideren los dos tipos de variables descritos, y puesto que la variable con unidad de medida transformada representa mejor la posición relativa de una determinada región o departamento, se le asignó un peso relativo mayor que a la variable con unidad de medida original; esto solo en los casos en que se utilizaron ambos tipos de variables.

\section{IX}

\section{Conclusiones}

La definición y alcances de la competitividad seguirán en desarrollo, quizá a la espera de consensos parciales ya sea en la definición o en los ámbitos de aplicación. Asimismo, no existe una metodología comprobada y difundida que permita saber el nivel de la calidad de los resultados de algún índice de competitividad, ya sea a nivel mundial o, como en este caso, regional. La dificultad radica en su condición de indicador relativo, es decir, que no determina qué región es competitiva y en cambio brinda una visión relativa de la competitividad de una determinada región con respecto a sus pares. De este modo, el ICR de un país es una herramienta o una guía para los negocios o políticas de Estado. Inclusive el papel de los índices de competitividad mundial, como una guía de desarrollo para los países, resulta duramente criticado al ser contrastado con los resultados de desarrollo de algunos países o regiones. Este es el caso de los índices anuales de competitividad mundial del WEF y el IMD que se publican todos los años, el del WEF desde el año 1979 y el del IMD desde el año 1988.

Desarrollar un índice de competitividad regional para un país constituye un verdadero esfuerzo por construir una herramienta que apoye su desarrollo. Cada aspecto considerado en su elaboración ha de ser el mejor; tomando en cuenta la revisión bibliográfica, el análisis crítico de otras experiencias, pero sobre todo, entendiendo claramente el concepto de competitividad regional que se quiere medir,
El segundo criterio de asignación de ponderaciones tiene que ver con la consistencia con la identificación de ventajas competitivas en la región. Son aquellas variables directamente vinculadas a aspectos fundamentales derivados de la definición de competitividad regional, como: i) productividad, ii) creatividad, iii) internacionalización, y iv) bienestar social, entre otros. Respecto de todas aquellas variables que tengan relación con estos conceptos se debe proceder a asignarles un peso relativo mayor que a las otras variables que la acompañen.

La disponibilidad y la calidad de la información a nivel subnacional en América Latina constituyen una importante barrera, derivada de los diferentes ordenamientos territoriales, grado de desagregación de la información, e importancia relativa de los factores o pilares con respecto a cada país, que podrían afectar los criterios de ponderación. Todo ello ligado al hecho de que la metodología se sustenta principalmente en información secundaria.

cual es elaborar una herramienta que aporte al desarrollo mediante la administración eficiente de los recursos de las regiones para el beneficio de sus pobladores y el incremento de la productividad empresarial.

Se identificaron los determinantes de la competitividad de las regiones a los que se denominó pilares: i) gobierno e instituciones, ii) desarrollo económico, iii) infraestructura productiva, iv) capital humano, y v) eficiencia de las empresas. Para cada uno de los pilares se identificaron cinco factores y sus variables que miden diversos aspectos de la competitividad regional. Estos constituyen un segundo y tercer nivel de desagregación que aporta al análisis que se puede realizar con los resultados que se obtengan.

El ICR de un país es un modelo específico desde el punto de vista estructural. Se compone de información estadística de fuentes secundarias y, en menor medida, de información primaria proveniente de una encuesta a empresarios de todas las regiones del país. De estas dos grandes fuentes de información se seleccionan los indicadores que dan forma a los cinco pilares que componen el ICR. Los indicadores seleccionados pasan por un proceso de estandarización a fin de consolidar las unidades de medida, para finalmente obtener tres tipos de resultados: i) el resultado global del ICR, ii) los resultados parciales del ICR para cada una de las regiones, y iii) los resultados parciales del ICR para cada uno de los cinco pilares. 


\section{Bibliografía}

América Economía (2008), Ranking infraestructura AE [en línea] http:// beta.americaeconomia.com/Multimedios/Otros/5503.pdf

Banco Mundial (2008), "Doing business" [en línea] http://www. doingbusiness.org/

Becker, G. (1964), Human Capital, Chicago, University of Chicago Press.

Blalock, H. (1970), Introducción a la investigación social, Buenos Aires, Amorrortu.

Bobadilla, P., del Águila, L. y M. de la L. Morgan (1998), Diseño y evaluación de proyectos de desarrollo, Lima, Pact-USAID.

CAF (Corporación Andina de Fomento) (2008), "Infraestructura" [en línea] http://www.CAF.com/view/index.asp? pageMS $=34366 \& \mathrm{~ms}=17$

Camagni, R. (2005), "El concepto de competitividad territorial", Economía urbana, R. Camagni, Barcelona, Antoni Bosch Editor.

CIDE (Centro de Investigación y Docencia Económicas) (2007), Competitividad de las ciudades mexicanas, México, D.F.

$\mathrm{CNC}$ (Consejo Nacional de Competitividad) (2008), Índice de Competitividad Regional, Lima.

D’Alessio, F. (2004), Administración y dirección de la producción. Enfoque estratégico y de calidad, México, D.F., Pearson.

Drucker, P. (1969), The Age of Discontinuity, Nueva York, Harper \& Row.

Esser, K. y otros (1996), "Competitividad sistémica: nuevo desafío para las empresas y la política", Revista de la Cepal, $\mathrm{N}^{\circ} 59$ (LC/G.1931-P), Santigo de Chile.

Foro Económico Mundial (2008), Informe de competitividad mundial 2008-2009, Ginebra.

(2006), Informe de competitividad mundial 2006-2007, Ginebra.

(2005a), Informe de competitividad mundial 2004-2005, Ginebra.

(2005b), Informe de competitividad mundial 2005-2006, Ginebra.

Garelli, S. (2006), "The competitiveness of nations: the fundamentals", IMD World Competitiveness Yearbook 2006 [en línea] http:// www.IMD.ch/documents/wcc/content/Fundamentals.pdf

Heritage Fundation (2008), 2009 Index of Economic Freedom [en línea] http://www.heritage.org/Index/

IMCO (Instituto Mexicano para la Competitividad) (2008), Competitividad estatal, Veracruz, México.

IMD (Instituto Internacional para el Desarrollo de la Capacidad de Gestión) (2008), IMD World Competitiveness Yearbook 2008, Lausana, Suiza.

Joy Way, R.L. (2004), Cómo establecer prioridades en las regiones del Perú: una propuesta de índice de competitividad regional sostenible, Piura, Perú, Consorcio de Investigación Económca y Social (CIES).

Keynes, J.M. (1936), Teoría general de la ocupación, el interés y el dinero, México, D.F., Fondo de Cultura Económica.

Kitson, M., R. Martin y P. Tyler (2004), "Regional competitiveness: an elusive yet key concept?", Regional Studies, vol. 38, No 9, Londres, Taylor \& Francis.

Krugman, P. (1998), Pop Internationalism, Cambridge, Massachusetts, The MIT Press.

(1994), "Competitiveness: a dangerous obsession", Foreign Affairs, vol. 73, N $\mathrm{N}^{\circ}$ 2, Nueva York, Council on Foreign Relations.

Larroulet, C. y F. Mochon (1995), Economía, Madrid, McGraw-Hill.
Lazarsfeld, P.F. (1958), "Evidence and inference in social research", American Academy of Arts \& Sciences, vol. 87, $\mathrm{N}^{\mathrm{o}} 4$, Cambridge, Massachusetts, American Academy of Arts \& Sciences.

León, J. (2003), ¿Cuál es el rol del Estado?, Revista de la Facultad de Ciencias Económicas de la UNMSM, año 5, № 15, Lima, Universidad Nacional Mayor de San Marcos.

Lucas, R. (1988), "On the mechanics of development planning", Journal of Monetary Economics, vol. 22, № 1, Amsterdam, Elsevier.

Malthus, T. (1798), First Essay on Population 1796, EnglewoodCliffs, Prentice Hall.

Mark, K. (1867), El capital: crítica de la economía política, México, D.F., Siglo XXI Editores.

PNUD (Programa de las Naciones Unidas para el Desarrollo) (2006), Informe sobre desarrollo humano del Perú, Lima.

Porter, M.E. (2001), ¿Dónde radica la ventaja competitiva de las naciones? Harvard Deusto Business Review, $\mathrm{N}^{\circ} 44$, Barcelona, Planeta. (2000), Estrategia competitiva: técnicas para el análisis de los sectores industriales y de la competencia, México, D.F., Compañía Editorial Continental.

(1999), Ser competitivo: nuevas aportaciones y conclusiones, Bilbao, Deusto.

(1991), La ventaja competitiva de las naciones, Buenos Aires, Javier Vergara Editor.

Prokopenko, J. (1972), Productivity Management. A Practical Handbook, Ginebra, Organización Internacional del Trabajo.

Ricardo, D. (1817), Principios de economía política y tributación, Madrid, Pirámide.

Sachs, J.D. y F.B. Larraín (1994), Macroeconomía en la economía global, México, D.F., Prentice Hall.

Schultz, T. (1961), "Investment in human capital", American Economic Review, vol. 51, № 1, Nashville, Tennessee, American Economic Review.

Schumpeter, J. (1962), Capitalismo, socialismo y democracia, México, D.F., Aguilar.

Sloan, A. (1963), My Years with General Motors.

Smith, A. (1776), "An inquiry into the nature and causes of the wealth of nations", The Harvard Classics, E.W. Charles, Nueva York, P.F. Colliers \& Son Corporation.

Solow, R. (1957), "El cambio técnico y la función de producción agregada", Economía del cambio tecnológico, N. Rosenberg, México, D.F., Fondo de Cultura Económica.

(1956), "A contribution to the theory of economic growth", Quarterly Journal of Economics, vol. 70, N ${ }^{\circ}$ 1, Cambridge, Massachusetts, The MIT Press.

SUBDERE (Subsecretaría de Desarrollo Regional y Administrativo) (2003), Informe de competitividad regional, Santiago de Chile, LOM Ediciones Ltda.

Tello, M.D. (2004), Los factores de competitividad en el Perú, Lima, Pontificia Universidad Católica del Perú.

Universidad Nacional de Colombia (2002), Sistema de indicadores de competitividad departamental, Bogotá, D.C., Centro de Investigaciones para el Desarrollo (CID).

Uzawa, H. (1965), "Optimal technical change in aggregative model of economic growth", International Economic Review, vol. 6, $\mathrm{N}^{\mathrm{o}}$ 1, Oxford, Blackwell Publishing.

Weber, M. (1905), The Protestant Ethic and the Spirit of Capitalism, Londres. 